\title{
TU/e emonownen

\section{Analysis of the Sampling Noise Cancellation Technique in a Track-and-Hold Amplifier}

\section{Citation for published version (APA):}

Li, H., Youssef, M., Shen, Y., Cantatore, E., \& Harpe, P. (2022). Analysis of the Sampling Noise Cancellation Technique in a Track-and-Hold Amplifier. In 2021 28th IEEE International Conference on Electronics, Circuits, and Systems, ICECS 2021 - Proceedings [9665546] Institute of Electrical and Electronics Engineers. https://doi.org/10.1109/ICECS53924.2021.9665546

DOI:

10.1109/ICECS53924.2021.9665546

Document status and date:

Published: 10/01/2022

\section{Document Version:}

Accepted manuscript including changes made at the peer-review stage

\section{Please check the document version of this publication:}

- A submitted manuscript is the version of the article upon submission and before peer-review. There can be important differences between the submitted version and the official published version of record. People interested in the research are advised to contact the author for the final version of the publication, or visit the $\mathrm{DOI}$ to the publisher's website.

- The final author version and the galley proof are versions of the publication after peer review.

- The final published version features the final layout of the paper including the volume, issue and page numbers.

Link to publication

\section{General rights}

Copyright and moral rights for the publications made accessible in the public portal are retained by the authors and/or other copyright owners and it is a condition of accessing publications that users recognise and abide by the legal requirements associated with these rights.

- Users may download and print one copy of any publication from the public portal for the purpose of private study or research.

- You may not further distribute the material or use it for any profit-making activity or commercial gain

- You may freely distribute the URL identifying the publication in the public portal.

If the publication is distributed under the terms of Article $25 \mathrm{fa}$ of the Dutch Copyright Act, indicated by the "Taverne" license above, please follow below link for the End User Agreement:

www.tue.nl/taverne

Take down policy

If you believe that this document breaches copyright please contact us at:

openaccess@tue.nl

providing details and we will investigate your claim. 


\title{
Analysis of the Sampling Noise Cancellation Technique in a Track-and-Hold Amplifier
}

\author{
Hanyue Li, Mina Youssef, Yuting Shen, Eugenio Cantatore and Pieter Harpe \\ Integrated Circuits Group, Eindhoven University of Technology, Eindhoven, The Netherlands \\ Email: h.li2@tue.nl
}

\begin{abstract}
The sampling noise cancellation (SNC) technique has been applied to different discrete-time circuits in recent literature, but the cost of this technique has not been well studied. This paper gives a detailed analysis of the SNC approach in a track-and-hold amplifier (THA). Closed-form expressions are derived for each noise source in the THA during the sampling phase and are verified by simulations. The analysis shows that while the sampling noise can be cancelled, the noise from the extra amplifier and switches can become dominant and even outweigh the benefits from SNC. Therefore, the SNC technique should be used with attention to the trade-offs, as described in this paper.
\end{abstract}

Index Terms - track-and-hold amplifier, kT/C noise, sampling noise cancellation

\section{INTRODUCTION}

The sampling process is ubiquitous in almost every discretetime circuit, such as switched-capacitor amplifiers and filters, analog-to-digital converters (ADC), etc. The well-known $k T / C$ noise associated with every sampling action has been regarded as a fundamental limit in such circuits, as a result of which every $6 \mathrm{~dB}$ improvement in signal-to-noise ratio (SNR) requires a quadrupled sampling capacitor, which leads to higher power consumption and larger chip area. Recently, a sampling noise cancellation (SNC) technique has been proposed and targeted to break this $k T / C$ noise limit [1]. It has been demonstrated in closed-loop sampling circuits [1]-[3] as well as open-loop track-and-hold circuits [4]-[6]. Despite the popularity of the SNC technique, its potential cost has not been examined in detail. This paper takes the track-andhold amplifier (THA) in which the SNC was initially proposed [1] as an example to investigate the trade-offs behind this technique.

The underlying principle of SNC is to treat the sampling noise as a time-varying offset for each sampling period. From this point of view, the methodology of auto-zeroing can be applied to cancel the $k T / C$ noise. Namely, the sampled noise voltage is first stored on a capacitor and then subtracted from the output. However, as auto-zeroing results in a higher noise floor, one can also expect that the SNC achieves the noise cancellation function at the expense of something else. This paper aims to answer this question by a thorough analysis of the SNC operation and it is organized as follows: Section II reviews the conventional THA and its sampling noise.

This work with project number 16594 is financed by the Dutch Research Council (NWO).
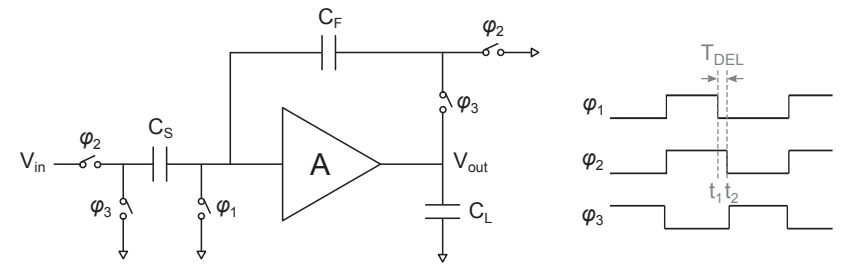

(a)

(b)

Fig. 1. Conventional THA (a) schematic (b) timing diagram.

Section III presents the THA with SNC and provides a detailed analysis for each noise source. Section IV shows the simulation results and explains the trade-offs in the SNC technique. Finally, Section V draws conclusions.

\section{Conventional THA}

Fig. 1 shows the conventional THA and its timing diagram. The THA is controlled by three clocks and operates in two phases: sampling phase $\left(\varphi_{1}\right.$ and $\left.\varphi_{2}\right)$ and amplification phase $\left(\varphi_{3}\right)$. The falling edge of $\varphi_{2}$ is delayed by $T_{D E L}$ compared to that of $\varphi_{1}$ in order to avoid signal-dependent charge injection from the sampling switches. The total noise of the THA is the summation of sampling noise from $\varphi_{1}$ and $\varphi_{2}$ phases and amplification noise from the $\varphi_{3}$ phase. This paper only focuses on the sampling noise, while the analysis of the amplification noise can be found in [7], and methods to cancel the thermal noise during the amplification phase are discussed in [8] and [9].

The sampling noise calculation of a conventional THA starts with the $\varphi_{1}$ switch noise. At the end of $\varphi_{1}$, a noise voltage $V_{n 1}$ is sampled on the right plate of $C_{S}$, as shown in Fig. 2(a). The total charge $Q_{\varphi 1}$ stored on $C_{S}$ and $C_{F}$ is:

$$
Q_{\varphi 1}=\left(V_{n 1}-V_{i n}\right) C_{S}+V_{n 1} C_{F} .
$$

During the $\varphi_{2}$ phase, the switches controlled by the $\varphi_{2}$ clock also generate noise. Assume that they generate instantaneous noise voltages $V_{n 2 L}$ and $V_{n 2 R}$ at the end of $\varphi_{2}$, as shown in Fig. 2(b). This gives rise to a voltage change $\Delta V_{n x}$ at the amplifier input node:

$$
\Delta V_{n x}=(1-\beta) V_{n 2 L}+\beta V_{n 2 R}
$$

where $\beta$ is $C_{F} /\left(C_{S}+C_{F}\right)$. However, since $Q_{\varphi 1}$ cannot change in this phase, no extra noise is sampled during $\varphi_{2}$. In phase 


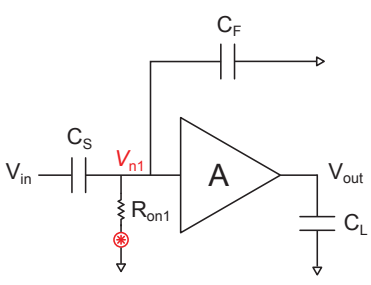

(a)

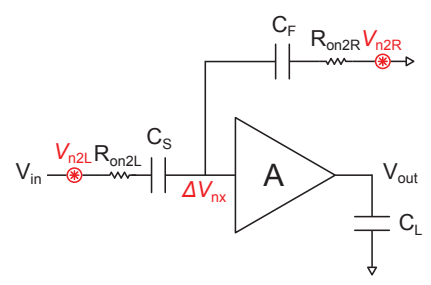

(b)
Fig. 2. Conventional THA noise model in (a) $\varphi_{1}$ phase and (b) $\varphi_{2}$ phase

$\varphi_{3}$, the loop is closed, and the amplifier input is forced to zero. Thus, the total charge $Q_{\varphi 3}$ on $C_{S}$ and $C_{F}$ is forced to:

$$
Q_{\varphi 3}=-V_{\text {out }} C_{F} .
$$

Due to charge conservation $\left(Q_{\varphi 3}=Q_{\varphi 1}\right)$, the amplifier output can be expressed as:

$$
V_{\text {out }}=V_{\text {in }} \frac{C_{S}}{C_{F}}-V_{n 1} \frac{C_{S}+C_{F}}{C_{F}} .
$$

It contains the amplified input signal as well as sampled noise. Since $V_{n 1}$ has a power of $k T /\left(C_{S}+C_{F}\right)$, where $k$ is the Boltzmann constant and $T$ is the absolute temperature, the total output-referred sampling noise power $\overline{V_{n, \text { out }}^{2}}$ is:

$$
\overline{V_{n, \text { out }}^{2}}=\frac{k T\left(C_{S}+C_{F}\right)}{C_{F}^{2}} .
$$

As expected, the sampling noise is expressed in a $k T / C$ format. This result is the same as the equation in [7] where the equipartition theorem is used for the noise calculation. In summary, the sampling noise in a conventional THA comes solely from the $\varphi_{1}$ switch, and it is not affected by the $\varphi_{2}$ switches and thus not dependent on the $T_{D E L}$ duration.

\section{THA With SAmpling Noise CANCELlation}

\section{A. SNC Principle}

Fig. 3 shows the THA structure with the SNC technique [1]. Compared to the conventional THA, a two-stage amplifier is used and an extra capacitor $C_{2}$ is inserted between the two amplifier stages. The first-stage amplifier $A_{1}$ has high bandwidth and low gain, while the second-stage amplifier $A_{2}$ has low bandwidth and high gain. The timing control for this THA is the same as that for the conventional case in Fig. 1(b).

To analyse the sampling noise, we can follow the same steps as in Section II. At the end of $\varphi_{1}$, the noise voltage $V_{n 1}$ is sampled on $C_{S}$ and $C_{F}$, and the total charge $Q_{\varphi 1}$ can still be expressed as (1). During $\varphi_{2}$, the amplified $V_{n 1}$ is stored on $C_{2}$, as shown in Fig. 4(a). When the amplifier is configured closed loop in $\varphi_{3}$, the right plate of $C_{2}$ will be forced to approximately zero thanks to the high gain from $A_{2}$. Since $C_{2}$ is floating in $\varphi_{3}$, the $A_{1}$ input will remain at $V_{n 1}$ instead of settling to zero, as illustrated in Fig. 4(b). Therefore, the total charge in $\varphi_{3}$ is:

$$
Q_{\varphi 3}=V_{n 1} C_{S}+\left(V_{n 1}-V_{\text {out }}\right) C_{F},
$$

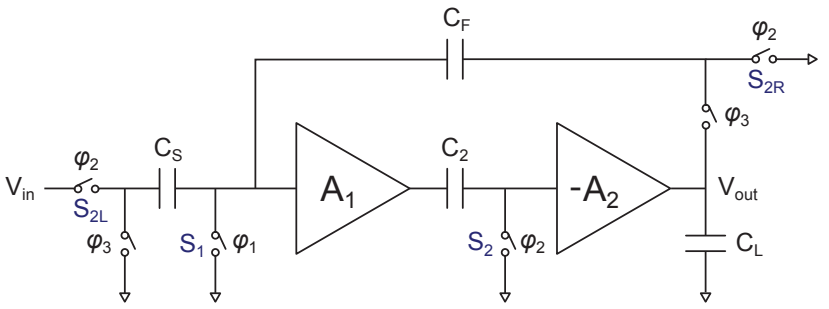

Fig. 3. THA with SNC technique.

and by combining this with (1) and noting that $Q_{\varphi 3}=Q_{\varphi 1}$, the final output becomes:

$$
V_{\text {out }}=V_{\text {in }} \frac{C_{S}}{C_{F}} .
$$

The sampling noise term $V_{n 1}$ is not present in (7), and therefore, the noise cancellation function is realized if all the other elements are ideal. However, if amplifier $A_{1}$ has a finite bandwidth, its output may not settle to $A_{1} V_{n 1}$ in the given time $T_{D E L}$ [1], resulting in incomplete noise cancellation. The remaining sampling noise can be written as [1]:

$$
\overline{V_{n, \text { out }}^{2}}=\frac{k T\left(C_{S}+C_{F}\right)}{C_{F}^{2}} \exp \left(\frac{-2 T_{D E L}}{R_{\text {out } 1} C_{2}}\right)
$$

where $R_{\text {out } 1}$ denotes the output impedance of the amplifier $A_{1}$. However, the above calculation only holds true if the noise from the $\varphi_{2}$ phase can be ignored. To guarantee an effective SNC, the other potential noise contributors (switches $S_{2}, S_{2 L}$, $S_{2 R}$ and amplifier $A_{1}$ ) need to be investigated carefully. These contributors in the THA have not been studied before, and thus this paper analyses these additional noise sources and verifies them by means of simulations.

\section{B. Noise from Switch $S_{2}$}

The noise generated from switch $S_{2}$ is shown in Fig. 4(c). At the end of $\varphi_{2}$, a noise voltage $V_{n 2}$ is sampled on the right plate of $C_{2}$. This makes the voltage kept on $C_{2}$ no longer $A_{1} V_{n 1}$ but $A_{1} V_{n 1}-V_{n 2}$. By repeating the noise calculation steps from the above sections, the $S_{2}$-induced output-referred noise can be obtained:

$$
\overline{V_{n, \text { out }}^{2}}=\frac{k T}{C_{2}} \cdot \frac{\left(C_{S}+C_{F}\right)^{2}}{A_{1}^{2} C_{F}^{2}} .
$$

\section{Noise from Switches $S_{2 L}$ and $S_{2 R}$}

To simplify the calculation, it is assumed that the ratio of the on-resistance of $S_{2 L}$ and $S_{2 R}$ satisfies $R_{o n 2 L} / R_{o n 2 R}=$ $C_{F} / C_{S}$, as shown in Fig. 4(d). Under this condition, the transfer function from each noise source to the amplifier input is a frequency-independent constant. This also indicates that the bandwidth of the noise seen at $V_{n x}$ is infinity. This noise will be filtered by the amplifier $A_{1}$ and leads to an amplifier output of $A_{1} V_{n x}^{\prime}$, where $V_{n x}^{\prime}$ is a band-limited signal. Like $V_{n 1}$ noise, the amplified $V_{n x}$ noise can also settle incompletely in the given $T_{D E L}$ duration. Following the above steps, the 


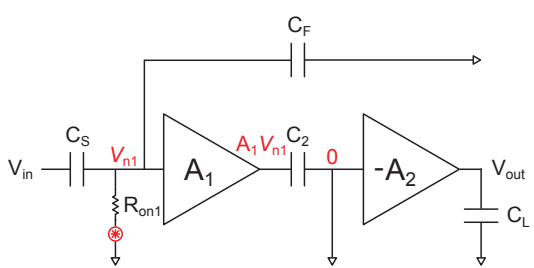

(a)

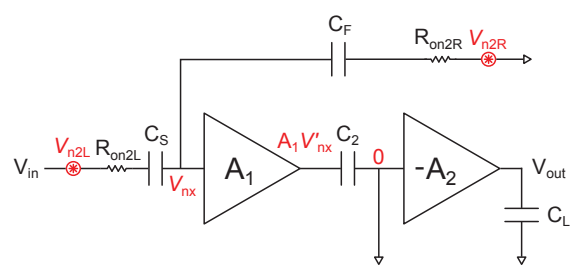

(d)

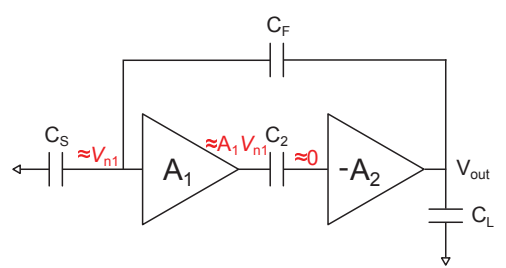

(b)

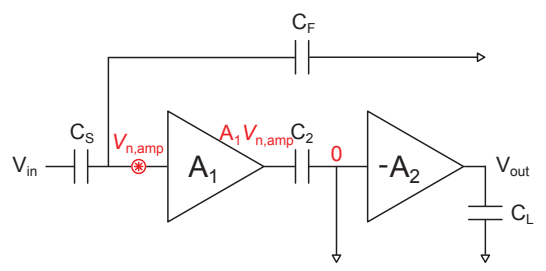

(e)

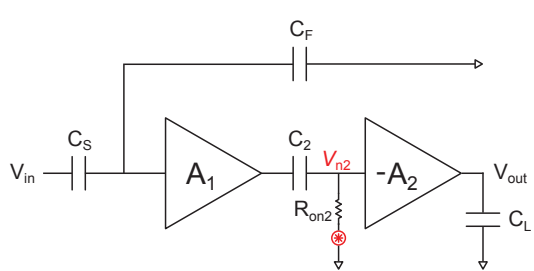

(c)

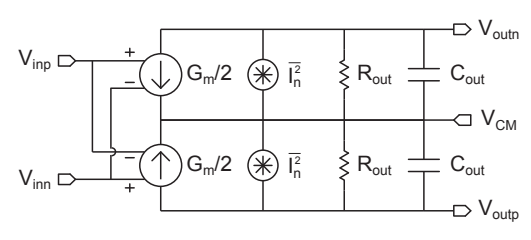

(f)

Fig. 4. THA with SNC technique in (a) sampling phase $\left(\varphi_{1}\right.$ and $\left.\varphi_{2}\right)$ and (b) amplification phase $\left(\varphi_{3}\right)$. Noise analysis of (c) switch $S_{2}$ (d) switches $S_{2 L}$ and $S_{2 R}$ (e) amplifier $A_{1}$. (f) Differential amplifier model.

output-referred noise caused by $S_{2 L}$ and $S_{2 R}$ can be shown to be:

$\overline{V_{n, \text { out }}^{2}}=\frac{k T\left(R_{\text {on } 2 L} C_{S}^{2}+R_{\text {on } 2 R} C_{F}^{2}\right)}{R_{\text {out } 1} C_{2} C_{F}^{2}}\left[1-\exp \left(\frac{-T_{D E L}}{R_{\text {out } 1} C_{2}}\right)\right]_{(10)}^{2}$.

\section{Noise from Amplifier $A_{1}$}

The noise from amplifier $A_{1}$ can be modeled as a noise voltage power spectral density $4 k T \gamma / G_{m 1}$, as shown in Fig. 4(e). This is filtered thanks to the finite bandwidth of $A_{1}$, resulting in a final output-referred noise of:

$$
\overline{V_{n, \text { out }}^{2}}=\frac{k T \gamma}{G_{m 1} R_{\text {out } 1} C_{2}} \cdot \frac{\left(C_{S}+C_{F}\right)^{2}}{C_{F}^{2}} .
$$

where $G_{m 1}$ is the transconductance of the amplifier $A_{1}$. Note that in this paper we only focus on noise contributions that are sampled in the network. Amplifiers $A_{1}$ and $A_{2}$ also add continuous-time noise during phase $\varphi_{3}$, but this is not included in the analysis, as they don't affect the actual sample.

\section{Simulation Results}

\section{A. Simulation Setup and Results}

To verify the above analysis, testbenches for both the conventional THA and a THA with SNC are created. The same two-stage amplifiers are used in these two testbenches. Each stage of the amplifier is built with the ideal components shown in Fig. 4(f). A differential configuration of the entire circuit is simulated, which doubles the output noise power. Each switch is modelled as an ideal switch in series with a resistor with $4 k T R$ noise. The transient noise simulation is performed using Cadence Spectre. The sampling period is 1 $\mu \mathrm{s}$, and the maximum noise frequency is set to be $5 \mathrm{GHz}$. The THA input is a DC signal of $10 \mathrm{mV}$. Other key parameters used in the simulation are summarized in Table I.

Fig. 5 shows the simulation results from the conventional THA without and with $\varphi_{2}$ switches noise. The simulated
TABLE I

Parameter VAlues in the Simulation

\begin{tabular}{|c|c|c|c|c|c|c|}
\hline Parameter & $C_{S}$ & $C_{F}$ & $C_{L}$ & $C_{2}$ & $\gamma$ & $T$ \\
\hline Value & $4 \mathrm{pF}$ & $1 \mathrm{pF}$ & $1 \mathrm{pF}$ & $1 \mathrm{pF}$ & 1 & $300 \mathrm{~K}$ \\
\hline Parameter & $G_{m 1}$ & $R_{\text {out } 1}$ & $C_{\text {out } 1}$ & $G_{m 2}$ & $R_{\text {out } 2}$ & $C_{\text {out } 2}$ \\
\hline Value & $10 \mathrm{mS}$ & $1 \mathrm{k} \Omega$ & $20 \mathrm{fF}$ & $1 \mathrm{mS}$ & $100 \mathrm{k} \Omega$ & $20 \mathrm{fF}$ \\
\hline Switches & \multicolumn{6}{|c|}{$R_{\text {on } 1}=R_{\text {on } 2}=R_{\text {on } 2 L}=10 \mathrm{k} \Omega, R_{\text {on } 2 R}=40 \mathrm{k} \Omega}$. \\
\hline
\end{tabular}

output noise matches the calculated value, and the noise is independent on $T_{D E L}$ as expected. Fig. 6 shows the simulated results from the THA with SNC. The individual noise sources are first simulated separately, as shown from Fig. 6(a) to Fig. 6(d). When one noise source is enabled, the other noise sources are disabled (e.g. by shorting the resistor which is in series with the ideal switch). It can be observed from Fig. 6(a) and Fig. 6(c) that the noise settles faster than what (8) and (10) predict. This is because we assumed a step response with zero initial condition when deriving (8) and (10). In practice, the amplifier $A_{1}$ output also tracks the switch noise during $\varphi_{1}$, so its settling does not start from the zero initial condition, and this shortens the time needed for complete settling. Fig. 6(e) shows the result when all the noise sources are included. The total noise is smaller than the addition of the individual noise values. This is because the switch on-resistance used in the simulation is relatively large, which reduces the bandwidth of some of the noise sources.

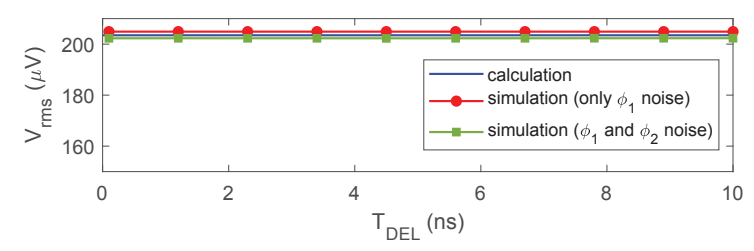

Fig. 5. Simulated output noise of the conventional THA. 


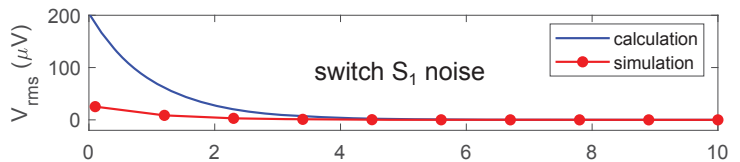

(a)

$\mathrm{T}_{\mathrm{DEL}}$ (ns)

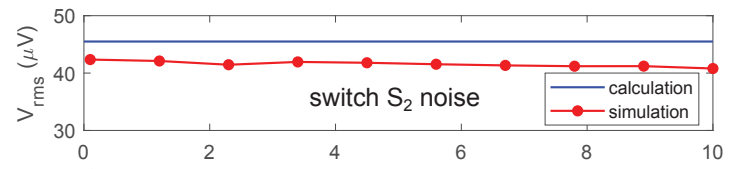

(b)

$T_{\text {DEL }}(n s)$

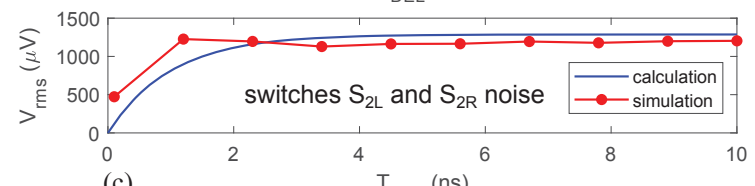

(c)

$T_{D E L}(n s)$

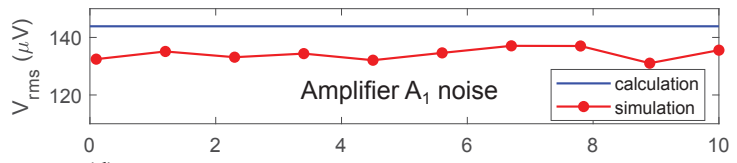

(d)

$\mathrm{T}_{\mathrm{DEL}}(\mathrm{ns})$

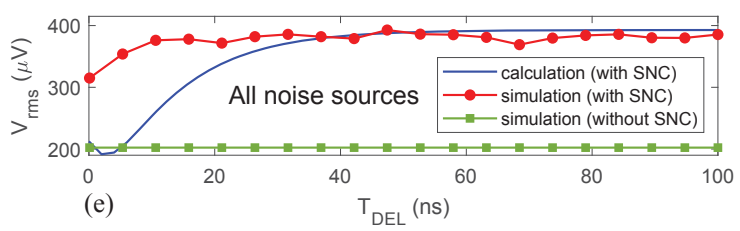

Fig. 6. Simulated output noise of the THA with SNC with only (a) $S_{1}$ noise (b) $S_{2}$ noise (c) $S_{2 L}$ and $S_{2 R}$ noise (d) amplifier $A_{1}$ noise and (e) all noise sources.
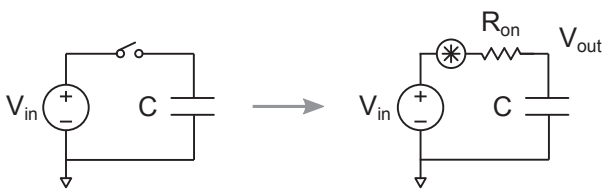

(a)
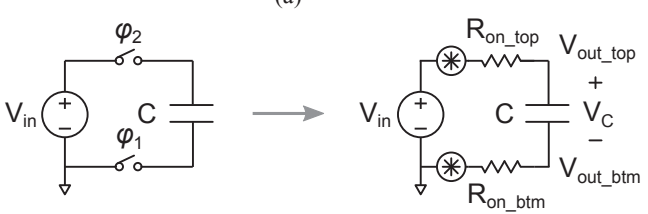

(b)

Fig. 7. (a) Ideal sampling and its noise model. (b) Bottom plate sampling and its noise model.

Fortunately, this reduction in the noise bandwidth can be easily incorporated into the existing equations by multiplying (9) by $R_{\text {on } 2} /\left(R_{\text {on } 2}+R_{\text {out } 1}\right)$ and replacing $R_{\text {out } 1}$ in (10) and (11) by $\left(R_{\text {on } 2}+R_{\text {out } 1}\right)$, respectively. The remaining difference between the simulation and calculation results in Fig. 6 is mainly due to the finite maximum noise frequency specified in the transient noise simulation (set to $5 \mathrm{GHz}$ ). This makes the simulated noise value in general lower than the calculated value. In summary, the theoretical calculations can be used as an accurate indication for the actual circuit noise.
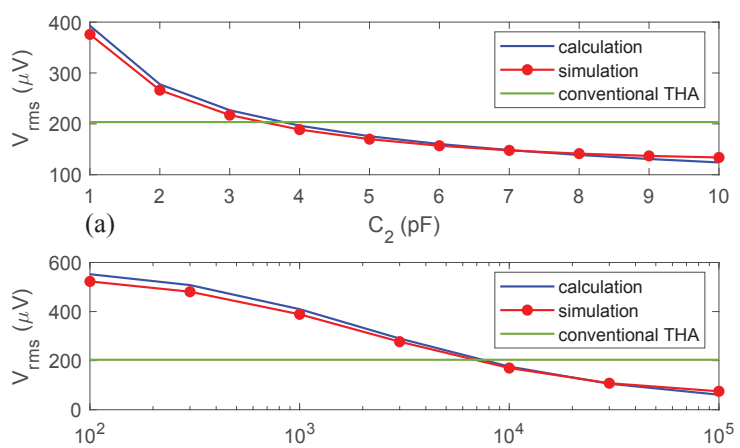

(b)

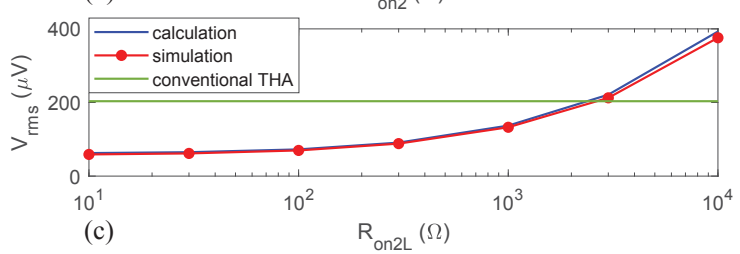

Fig. 8. Simulated output noise of the THA with SNC by (a) sweeping $C_{2}$ $\left(R_{o n 1}=R_{o n 2}=R_{o n 2 L}=10 \mathrm{k} \Omega, R_{o n 2 R}=40 \mathrm{k} \Omega\right)(\mathrm{b})$ sweeping $R_{o n 2}$ $\left(C_{2}=5 \mathrm{pF}, R_{\text {on } 1}=R_{\text {on } 2 L}=10 \mathrm{k} \Omega, R_{\text {on } 2 R}=40 \mathrm{k} \Omega\right)$ (c) sweeping $R_{\text {on } 2 L}$ $\left(C_{2}=1 \mathrm{pF}, R_{\text {on } 1}=R_{\text {on } 2}=10 \mathrm{k} \Omega, R_{\text {on } 2 R}=4 R_{\text {on } 2 L}\right)$.

\section{B. Discussion}

As shown in Fig. 6(a), the SNC technique can indeed cancel the original noise from the sampling switch $S_{1}$. However, the total sampled noise of the THA with SNC as shown in Fig. 6(e) is even higher than that of the conventional THA in this particular simulation example. Putting aside the intricate equations, we can interpret this behaviour intuitively from two aspects.

First, the actual sampling moment is different in these two scenarios. In the conventional THA case, the actual sampling happens at $t_{1}$ in Fig. 1(b). This means that all the noise injected at moment $t_{1}$ will be maintained in the system while the noise at moment $t_{2}$ is not critical. However, the SNC moves the actual sampling moment to $t_{2}$ [4]. Although it could cancel the sampling noise at $t_{1}$, more noise sources are present at $t_{2}$ due to the extra amplification and sampling process on $C_{2}$. This approach thus transfers the noise origin from one component to another one, instead of removing it.

Second, the cancelled noise is not actually the classic $k T / C$ noise that is shown in Fig. 7(a). Due to the bottom plate sampling, switches are needed at both the top and bottom plates of the capacitor in Fig. 7(b). In this case, only the voltage across the capacitor $\left(V_{C}\right)$ has the noise power of $k T / C$. The plate voltages $V_{\text {out_top }}$ and $V_{\text {out_btm }}$ are correlated and have a much larger noise bandwidth. The SNC technique only cancels the noise on $V_{\text {out_btm }}$ instead of cancelling the real $k T / C$ noise across the sampling capacitor. The analysis of SNC in prior art [1][4] assumes a zero $R_{\text {on_top }}$ and ignores its noise contribution.

To make the SNC technique effective, we need to make sure that the sum of the noise from (8) to (11) is smaller than (5). For simplicity, considering the noise from $S_{2 L}$ and $S_{2 R}$ 
is dominant in Fig. 6, we can set the noise in (10) less than that in (5) as an example. Note that $R_{\text {out } 1}$ in (10) needs to be replaced by $\left(R_{\text {on } 2}+R_{\text {out } 1}\right)$ as explained in Section IVA. This yields:

$$
(1+\lambda)\left(R_{\text {on } 2}+R_{\text {out } 1}\right) C_{2}>\left(R_{\text {on } 2 L}+\lambda^{2} R_{\text {on } 2 R}\right) C_{S} .
$$

where $\lambda=C_{F} / C_{S}$ and $T_{D E L}$ is assumed long enough. Several design choices can be made to satisfy (12), as shown in Fig. 8. First, $C_{2}$ can be made larger at the expense of higher $A_{1}$ power. But if $C_{2}$ is designed to be even larger than $C_{S}$, this will be against our initial intention to reduce the total sampling capacitance. Second, the $R_{\text {on } 2}$ and $R_{\text {out } 1}$ can be increased to reduce the noise bandwidth, but this may lead to long $T_{D E L}$ and is thus suboptimal. Third, $R_{o n 2 L}$ and $R_{o n 2 R}$ should be sufficiently small. However, small $R_{o n 2 L}$ also implies that a low output impedance from the driving stage in front of the THA (not shown in the figures) is needed.

For a given bandwidth requirement, the sampling capacitor $C_{S}$ size can be reduced thanks to $\mathrm{SNC}$, and this can relax the input driver specification from the speed perspective. But the on-resistance of $S_{2 L}$ and the driver output impedance still need to be kept low due to the noise restrictions in (12). This can instead make the driver consume more power due to the low noise spectral density requirement. Therefore, the input driver needs to be designed carefully with the strict noise budget as well as the speed specification.

\section{Conclusion}

This paper examines the principle of the SNC technique in a THA thoroughly. All the noise sources in the sampling phase are included in the analysis, and the theoretical calculations are verified by simulations. The trade-offs between switch onresistance, amplifier power, driver strength, total capacitance, and the final noise power are discussed. Overall, the SNC technique can indeed reduce sampling noise, but it comes at the cost of increased power or area inside the THA, or increased power in the driving stage in front of the THA.

\section{REFERENCES}

[1] R. Kapusta, H. Zhu and C. Lyden, "Sampling circuits that break the kT/C thermal noise limit," IEEE J. of Solid-State Circuits, vol. 49, no. 8, pp. 1694-1701, Aug. 2014.

[2] J. Xu, A. T. Nguyen, D. K. Luu, M. Drealan and Z. Yang, "Noise optimization techniques for switched-capacitor based neural interfaces," IEEE Trans. on Biomed. Circuits and Syst., vol. 14, no. 5, pp. 10241035, Oct. 2020

[3] C. Okada et al., "A high-speed back-illuminated stacked CMOS image sensor with column-parallel kT/C-cancelling S\&H and Delta-Sigma ADC,' in IEEE Int. Solid-State Circuits Conf. (ISSCC), 2021, pp. 116 118.

[4] J. Liu, X. Tang, W. Zhao, L. Shen and N. Sun, "A 13-bit 0.005-mm² 40$\mathrm{MS} / \mathrm{s}$ SAR ADC with kT/C noise cancellation," IEEE J. of Solid-State Circuits, vol. 55, no. 12, pp. 3260-3270, Dec. 2020.

[5] S. Li, "A kT/C-noise-cancelled noise-shaping SAR ADC with a dutycycled amplifier," in IEEE Int. Midwest Symposium on Circuits and Systems (MWSCAS), 2020, pp. 758-761.

[6] T. -H. Wang, R. Wu, V. Gupta and S. Li, "A 13.8-ENOB 0.4pF-C ${ }_{\text {IN }} 3^{\text {rd }}$ order noise-shaping SAR in a single-amplifier EF-CIFF structure with fully dynamic hardware-reusing kT/C noise cancelation," in IEEE Int Solid-State Circuits Conf. (ISSCC), 2021, pp. 374-376.

[7] B. Murmann, "Thermal noise in track-and-hold circuits: analysis and simulation techniques," IEEE Solid-State Circuits Magazine, vol. 4, no. 2, pp. 46-54, Spring 2012.

[8] B. D. Sahoo and A. Inamdar, "Thermal-noise-canceling switchedcapacitor circuit," IEEE Trans. on Circuits and Syst. II: Express Briefs, vol. 63, no. 7, pp. 628-632, Jul. 2016.

[9] C. Ravi, D. James, V. Sarma, B. D. Sahoo and A. Inamdar, "Thermal noise canceling pipelined ADC," in IEEE Int. Symposium on Circuits and Systems (ISCAS), 2017, pp. 1-4. 